\title{
Rentabilidad y rendimiento de girasol en función del tipo de suelo, nitrógeno y biofertilizante
}

\author{
Sunflower profitability and grain yield as function of soil type, nitrogen and biofertilizer
}

\author{
Y.R. Arenas-Julio', J.A.S. Escalante-Estrada' ${ }^{1}$ C. Aguilar-Carpio ${ }^{3 *}$, M.T. Rodriguez-Gonzalez ${ }^{1}$ y E. Sosa-Montes ${ }^{2 *}$ \\ Colegio de Postgraduados. Campus Montecillo. Km 36.5 Carretera México-Texcoco, CP. 56230. Montecillo, Texcoco, Estado \\ de México, México. \\ Departamento de Zootecnia, Universidad Autónoma Chapingo. Carretera Federal México-Texcoco Km 38.5, CP. 56230. \\ IDAGRO S. de R. L. de C. V. Carretera Yautepec-Tlayacapan S/N. Col. Puente Pantitlán. Tlayacapan, Mor. C. P. 62545.
}

\section{RESUMEN}

El objetivo de este estudio fue determinar la influencia del tipo de suelo, nitrógeno y biofertilizante sobre la fenología, biomasa total, rendimiento de grano y sus componentes en el cultivo de girasol. El estudio se realizó en el Colegio de Postgraduados, donde se sembró el girasol cv. Victoria. Los tratamientos en estudio fueron dos tipos de suelo en los cuales se aplicó dos niveles de nitrógeno, con y sin biofertilizantes. Se evaluó la fenología de la planta, biomasa total, índice de cosecha, rendimiento y sus componentes. Para los suelos en estudio se encontró que la fertilización nitrogenada y el biofertilizante no modificaron el tiempo a ocurrencia de las etapas fenológicas, pero sí se observó un cambio en la biomasa total y rendimiento de grano, donde la mayor producción se registró con la aplicación de nitrógeno a 100 kg ha $^{-1}$ y la inoculación del biofertilizante, lo que a su vez generó el mayor ingreso neto, en el suelo con la salinidad más baja. Palabras clave: Helianthus annuus, biomasa total, fertilización, salinidad.

\section{ABSTRACT}

The objective of this study was to determine the influence of soil type, nitrogen and biofertilizer on phenology, total biomass, grain yield and its components on sunflower cultivation. At the Postgraduates College, the study was carried out, where the sunflower cv Victory was sowed. The treatments under study were two types of soil with two nitrogen levels, with and without biofertilizers. The plant's phenology, total biomass, harvest index, grain yield and its components were evaluated. For the soils under study, nitrogen fertilization and the biofertilizer were found not to affect the phenological stages, but a change in total biomass and grain yield was observed, where the highest production and net income was recorded with the application of nitrogen at $100 \mathrm{~kg} \mathrm{ha}^{-1}$ and biofertilizer inoculation, in the soil with the lowest salinity.

Key words: Helianthus annuus, total biomass, fertilization, salinity.

\section{INTRODUCCIÓN}

El girasol (Helianthus annuus L.), es uno de los cultivos oleaginosos más importantes para la producción de aceite,

*Autor para correspondencia: Cid Aguilar Carpio

Correo electrónico: aguilar.cid@colpos.mx

Recibido: 07 de mayo de 2020

Aceptado: 12 de agosto de 2020

además es considerado como uno de los aceites con mayores beneficios para la salud, por su alto contenido de ácidos grasos poliinsaturados (Werteker et al., 2010). En México en los últimos años ha tomado importancia debido a que es una oleaginosa con gran potencial aceitera. Para el 2018 la producción nacional de girasol fue de 9,263 t en una superficie cosechada de 6,629 ha, con un rendimiento promedio de $1.39 \mathrm{t} \mathrm{ha}^{-1}$, donde los principales estados productores fueron Sonora, Zacatecas, Guanajuato, Jalisco y Estado de México (SIAP, 2018).

Con el uso de prácticas agrícolas adecuadas se provee a los cultivos una mayor expresión del rendimiento, dentro de dichas prácticas destacan la preparación del suelo, densidad de población y fertilización. El rendimiento de girasol es controlado por factores, como la selección de híbridos, una adecuada tecnología, el manejo de prácticas agrícolas especialmente en la densidad óptima de población y el uso de fertilizantes (nitrógeno) (Ishfaq, 2009). Investigaciones anteriores que se han desarrollado con girasol, han demostrado que con aplicaciones de fertilizante nitrogenado se han incrementado el rendimiento de semilla por unidad de superficie, la producción de materia seca, número de semiIlas $\mathrm{m}^{-2}$ y área del capítulo (Ahmed y El-Araby, 2012; Ali et al., 2012; Nasim et al., 2012). Un indicador importante respecto al uso del nitrógeno es su eficiencia agronómica, que indica el incremento en el rendimiento económico de un cultivo por unidad de nitrógeno aplicado (Ali et al., 2012).

Las características del suelo como su textura, mineralogía, estructura, materia orgánica son en gran medida responsables de como el suelo afecta el rendimiento en cultivos. Un problema que limita el crecimiento y desarrollo de las plantas es la salinidad del suelo, el origen de estos tipos de suelos pude ser muy diverso pudiendo ser de manera general o antrópico. La salinidad del suelo es un problema que se incrementa año con año en las regiones áridas y semiáridas del mundo como consecuencia de una baja precipitación y un mal manejo del agua de riego y los fertilizantes. La salinidad y la sequía son dos de los factores limitativos ambientales que afectan el establecimiento y desarrollo de las especies, así como la producción agrícola (Martínez et al., 2011).

Por otro lado, los fertilizantes microbiológicos son considerados una parte importante de las prácticas agrí- 
colas sostenibles, con entradas de bajo costo; fijación de nitrógeno, solubilización de fosfato, potasa y de movilización de microorganismos en la planta (Mishra y Dadhich, 2010). Los biofertilizantes contienen microorganismos específicos en formas concentradas, derivados de la zona radicular del suelo, en donde establecen una simbiosis mutualista con las raíces de la mayoría de las plantas; los cuales ayudan a mejorar el crecimiento gracias al sistema de hifas que se desarrollan fuera de la raíz y que permiten una mayor exploración del suelo al incrementar la captación de nutrimentos poco móviles, resistencia y/o tolerancia de la planta a la sequía y salinidad (Tapia et al., 2010). Para lograr una producción sostenible de girasol es necesario complementar la fertilización química con formas biológicas, para obtener en lo posible un impacto en la producción y en la conservación del ambiente. Las bacterias y hongos son capaces de proveer a la planta diferentes nutrientes en forma asimilable. Esto puede ocurrir mediante diferentes procesos, por ejemplo, la inoculación de las plantas con micorrizas, que contribuye a incrementar la absorción de agua, solubilizar los minerales y transformar el fósforo que se encuentra en el suelo, formando compuestos estables, en formas disponibles para la planta (Madhurendra et al., 2009; Zarabi et al., 2011; Ahmed y El-Araby, 2012). Múltiples estudios han revelado que los biofertilizantes influyen en el crecimiento y producción de los cultivos. Al respecto, Sotelo et al. (2016) y Mena et al. (2018) observaron una mayor producción en la biomasa, rendimiento y diámetro del capítulo con el uso de biofertilizantes en el cultivo de girasol. Por lo cual el objetivo del presente estudio fue determinar la influencia del tipo de suelo, nitrógeno y biofertilizante sobre la fenología, biomasa total, rendimiento de grano y sus componentes (peso de 100 granos y área del capítulo) en el cultivo de girasol.

\section{MATERIALES Y MÉTODOS}

El estudio se estableció en dos sitios del Colegio de Postgraduados, Montecillo, Estado de México ( $19^{\circ} 29^{\prime}$ norte; $98^{\circ} 54^{\prime}$ oeste y $2,250 \mathrm{msnm}$ ), bajo condiciones de temporal. El clima es de tipo BS1 que corresponde al menos seco de los áridos con lluvias en verano, una temperatura media anual de $14.6^{\circ} \mathrm{C}$ y precipitación media anual de $500 \mathrm{~mm}$ (García, 2004). En el mes de mayo de 2012, se sembró el girasol cv. Victoria (aceitero) en una densidad de 80,000 plantas ha-1. Los tratamientos fueron: dos tipos de suelo, los cuales presentaron las siguientes características: suelo 1 (S1), textura franco arenoso, $\mathrm{pH}$ de 7.1 y CE de $0.49 \mathrm{dS} \mathrm{m}^{-1}$, suelo 2 (S2), textura franco arcilloso limoso, $\mathrm{pH}$ de 7.8, CE de $1.75 \mathrm{dS} \mathrm{m}^{-1}$; dos niveles de nitrógeno de 0 (N0) y $100 \mathrm{~kg} \mathrm{ha}^{-1}$ (N100), los cuales se aplicaron de forma fraccionada, la mitad al momento de la siembra y el resto a los 30 días, como fuente de nitrógeno se utilizó urea ( $46 \%$ de N); con y sin biofertilizantes, el cual consistió en la inoculación a la semilla de bacterias fijadoras de nitrógeno Azospirillum brasilense $(0.4 \mathrm{~kg})$ y hongos micorrízico Glomus intraradices $(1 \mathrm{~kg})$, las cuales fueron mezcladas con agua y adherente. El diseño experimental fue bloques completos al azar con arreglo de parcelas subdivididas y cuatro repeticio- nes. Durante el desarrollo del estudio se registró la temperatura máxima, mínima, evaporación y precipitación, los datos fueron proporcionados por la estación agrometeorológica del Colegio de Postgraduados. La evapotranspiración del cultivo $\left(E T c, \mathrm{~mm} \mathrm{~d}^{-1}\right)$ se calculó mediante la ecuación:

$$
\mathrm{ETC}=\mathrm{Ev} \times \mathrm{Ke} \times \mathrm{Kc},(\text { Doorenbos y Pruitt, 1986) }
$$

Donde: $\mathrm{Ev}=$ evaporación del tanque $\mathrm{A} ; \mathrm{Ke}=$ coeficiente para el evaporímetro (0.6); y Kc = coeficiente del cultivo (0.8 para girasol). Así mismo se calculó las unidades calor (UC, ${ }^{\circ} \mathrm{C}$ d), mediante el método residual de Flores et al. (1985), el cual es descrito por la siguiente ecuación:

$$
\text { UC }=\left(\frac{\text { Tmáx+Tmín }}{2}-\mathrm{TB}\right.
$$

Dónde: Tmáx = Temperatura máxima diaria $\left({ }^{\circ} \mathrm{C}\right)$, Tmín $=$ Temperatura mínima diaria $\left({ }^{\circ} \mathrm{C}\right)$ y $\mathrm{TB}=$ Temperatura base, considerada como $8{ }^{\circ} \mathrm{C}$ (Sadras y Hali, 1988). Las etapas fenológicas registradas fueron: días a emergencia (E), floración (R5) y madurez fisiológica (R9) (Schneiter y Miller, 1981). A la cosecha se registró la biomasa total (BT) (peso de receptáculo, tallo y hojas + peso del grano), índice de cosecha (IC), rendimiento de grano (RG) y sus componentes, como área del capítulo (AC) y peso de 100 semillas (P100S). A las variables en estudio se les aplicó un análisis de varianza y se aplicó la prueba de comparación de medias Tukey $(P \leq 0.05)$ con el paquete estadístico SAS versión 9.1 (SAS, 2004).

Para el análisis económico se estimaron los costos arrojados por cada tratamiento utilizando las siguientes ecuaciones (Bueno et al., 2005):

Costo total (CT), es la suma de los costos fijos (CF) y variables (CV).

$$
\mathrm{CT}=(\mathrm{CF}+\mathrm{CV})
$$

Ingreso total (IT), se calculó con la siguiente fórmula:

$\mathrm{IT}=$ Py $\mathrm{Y}$

Donde: $\mathrm{Py}=$ precio del producto ( $\$ 20.00$ para ese año); $\mathrm{Y}=$ producción $\mathrm{ha}^{-1}$.

Ingreso neto (IN), es la diferencia del costo total (CT) y el ingreso total (IT).

IN $=(\mathrm{IT}-\mathrm{CT})$

Ganancia por peso invertido (GPI), se obtuvo dividiendo el ingreso neto (IN) entre el costo total (CT).

$$
\mathrm{GPI}=(\mathrm{IN} / \mathrm{CT})
$$

\section{RESULTADOS Y DISCUSIÓN \\ Elementos del clima y fenología}

En la Figura 1, se presentan los datos de temperatura máxima (Tmax), mínima (Tmin) promedio decenal y la precipitación suma decenal, durante el ciclo del cultivo se observó que el promedio decenal durante la estación de crecimiento de Tmax fluctuó entre 34 y $22^{\circ} \mathrm{C}$ y la Tmin de 12 a $-2{ }^{\circ} \mathrm{C}$. Las condiciones climáticas de temperaturas fueron apropiadas para el desarrollo del cultivo del girasol (Escalante et al., 2015). La temperatura más alta ocurrió en la etapa vegetativa del cultivo en los meses de mayo y junio, posteriormente, 
Figura 1. Temperatura máxima, mínima (media decenal) y precipitación (suma decenal) durante el ciclo de cultivo de girasol. $\mathrm{E}=$ Emergencia, R5 = Floración y R9 = Madurez fisiológica.

Figure 1. Maximum, minimum temperature (average decenal) and precipitation (sum decenal) during the sunflower growing cycle. $E=$ Emergency, R5 = Flowering and R9 = Physiological maturity.

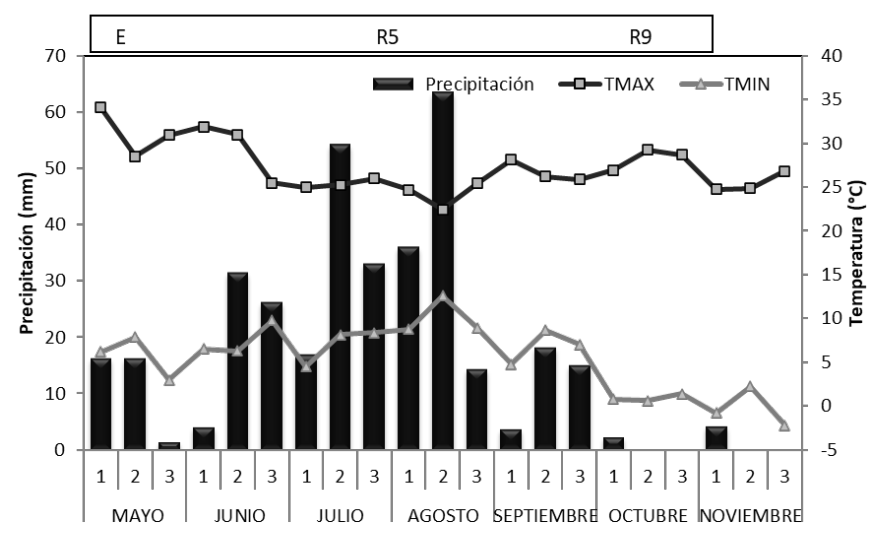

disminuyó conforme el ciclo de cultivo avanzó, debido principalmente al incremento en la precipitación (386 mm), donde la más alta se observó en el mes de agosto a inicios de la floración, sin embargo, se redujo en etapa de llenado de grano, lo cual indica que al final del ciclo el cultivo presentó un déficit hídrico, que pudo afectar el rendimiento (Escalante y Rodríguez, 2010).

La ocurrencia de las etapas fenológicas fue similar para todos los tratamientos. Esto, se pudo deber a que la nutrición en el suelo fue suficiente para que el cultivo no modificara la ocurrencia de las etapas fenológicas durante el desarrollo del estudio. Así, para ambos suelos la E se presentó a los 8 dds, el periodo de R5 ocurrió a los 68 dds y la madurez fisiológica se presentó a los 120 días (Figura 1). Estos resultados concuerdan parcialmente con los reportados por Escalante y Rodríguez (2010) al evaluar la fenología de girasol en tres niveles de salinidad del suelo y en agrosistemas (girasol-frijol), en donde la E se presentó a los 8 dds, el periodo de R5 ocurrió a los 80 dds y R9 a los 130 dds.

\section{Unidades Calor y evapotranspiración}

La acumulación de unidades calor (UC) en relación con la ocurrencia de las etapas fenológicas del cultivo se presentan en la Figura 2. El requerimiento térmico del girasol fue similar entre tratamientos. De esta manera, la acumulación de UC fue de $125^{\circ} \mathrm{C}, 705^{\circ} \mathrm{C}$ y $1,551^{\circ} \mathrm{C}$ durante las etapas $\mathrm{E}$, $\mathrm{R} 5$ y MF, respectivamente, datos similares fueron reportados por Morales et al. (2006) al evaluar girasol en cultivo asociado con frijol (Phaseolus vulgaris. L). El modelo para estimar las UC para el genotipo y condiciones del presente estudio fue UC $=22.9 \mathrm{dds}, \mathrm{R}^{2}=0.99^{* *}$. La ETc del girasol también fue similar entre tratamientos. En la Figura 2 se muestra que la ETc acumulada para las etapas $\mathrm{E}, \mathrm{R} 5$ y MF fue de $18 \mathrm{~mm}, 139 \mathrm{~mm}$ y $377 \mathrm{~mm}$, respectivamente.

En la Figura 2, que relaciona la evapotranspiración acumulada del cultivo (ETc), se observa que el periodo de siembra a emergencia, la ETc fue de $18 \mathrm{~mm}$, en el periodo

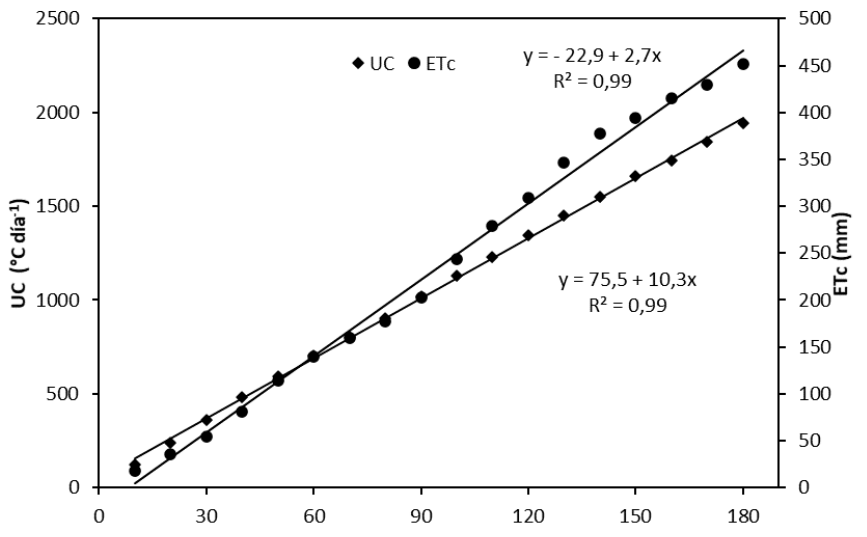

Figura 2. Unidades calor (UC) y Evapotranspiración (ETC) acumuladas de girasol cv. Victoria. E = Emergencia, R5 = Floración, R9 = Madurez fisiológica. Figure 2. Accumulated heat units (UC) and Evapotranspiration (ETC) from sunflower cv. Victory. E = Emergency, R5 = Flowering, R9 = Physiological maturity.

de emergencia a antesis la ETc fue de $121 \mathrm{~mm}$ y de antesis a madurez fisiológica fue de $256 \mathrm{~mm}$. La ETc acumulada durante todo el ciclo del cultivo fue de $377 \mathrm{~mm}$. Así mismo Morales et al. (2006), al evaluar el girasol cv. Victoria en un clima templado encontraron que de la siembra a emergencia la ETc fue de $15 \mathrm{~mm}$, de emergencia a antesis fue de $185 \mathrm{~mm}$ y de antesis a madurez fisiológica fue de $80 \mathrm{~mm}$. Teniendo una ETc acumulada durante todo el ciclo del cultivo de 280 $\mathrm{mm}$.

En el Tabla 1, se presenta el análisis de varianza de los factores de estudio y sus interacciones, en el cual muestra que la biomasa total (BT) y rendimiento de grano (RG) presentaron diferencias significativas para el suelo (S), nitrógeno $(\mathrm{N})$, biofertilizante $(\mathrm{BIO}), \mathrm{S} \times \mathrm{N}, \mathrm{S} \times \mathrm{BIO}$ y N x BIO; mientras que en el IC se observó una nula significancia en las interacciones $\mathrm{S} \times \mathrm{BIO}$ y $\mathrm{N} \times \mathrm{BIO}$. Con respecto al P100S la significancia se registró en el factor $\mathrm{N}$, BIO y la interacción $\mathrm{S} \times \mathrm{BIO}$, finalmente para AC no presentaron diferencias significativas las interacciones $\mathrm{S} \times \mathrm{N}, \mathrm{S}$ x BIO y $\mathrm{N}$ x BIO.

Tabla 1. Biomasa total (BT), índice de cosecha (IC), rendimiento de grano $(\mathrm{RG})$, peso de 100 semillas (P100S) y área de capítulo (AC), en función al tipo de suelo $(\mathrm{S})$, nitrógeno $(\mathrm{N})$ y biofertilizante $(\mathrm{BIO})$.

Table 1. Total biomass (BT), harvest index (IC), grain yield (RG), 100 seeds weight (P100S) and chapter area (AC), as a function of soil type (S), nitrogen (N) and biofertilizer (BIO).

\begin{tabular}{|c|c|c|c|c|c|}
\hline FACTOR & $\begin{array}{c}\text { BT } \\
\mathrm{g} \mathrm{m}^{-2} \\
\end{array}$ & $\begin{array}{l}\text { IC } \\
\% \\
\end{array}$ & $\begin{array}{c}\text { RG } \\
\mathrm{g} \mathrm{m}^{-2} \\
\end{array}$ & $\begin{array}{c}\text { P100S } \\
\text { g } \\
\end{array}$ & $\begin{array}{l}\mathrm{AC} \\
\mathrm{cm}^{2}\end{array}$ \\
\hline$S$ & $* *$ & $* *$ & $* *$ & NS & $* *$ \\
\hline$N$ & $* *$ & $* *$ & $* *$ & $* *$ & $* *$ \\
\hline $\mathrm{BIO}$ & $* *$ & $*$ & * & * & $* *$ \\
\hline $\mathrm{S} \times \mathrm{N}$ & * & $* *$ & * & NS & NS \\
\hline $\mathrm{S} \times \mathrm{BIO}$ & * & NS & * & * & NS \\
\hline $\mathrm{N} \times \mathrm{BIO}$ & * & NS & * & NS & NS \\
\hline $\mathrm{S} \times \mathrm{N} \times \mathrm{BIO}$ & * & * & NS & NS & $*$ \\
\hline
\end{tabular}

${ }^{*}=\mathrm{P} \leq 0.05,{ }^{* *}=\mathrm{P} \leq 0.01, \mathrm{NS}=$ Diferencias no significativas a $\mathrm{P}>0.05$. 


\section{Suelo}

La producción de BT y RG más alta (Tabla 2) se ob-

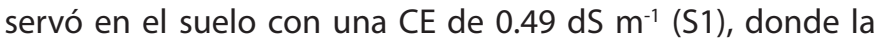
producción se incrementó en 17 y $21 \%$ respecto a S2, el cual presenta una CE de $1.75 \mathrm{dS} \mathrm{m}^{-1}$; tendencias similares se observó en el IC, P100S y el AC donde los mayores datos se encontraron en $\mathrm{S} 1$ por registrar una conductividad eléctrica más baja en comparación a S2. Dos-Santos et al. (2017) señalan que la reducción en la producción de las plantas en estrés salino se explica por exceso de acumulación de ciertos iones en los tejidos vegetales, disminución en el potencial osmótico de la solución del suelo, posible toxicidad iónica e inestabilidad nutricional. Al respecto Escalante y Rodríguez (2010), al evaluar girasol en suelos con diferentes grados de salinidad, encontraron que la producción de BT y RG se vio afectada en suelos con mayor salinidad.

Tabla 2. Biomasa total (BT), índice de cosecha (IC), rendimiento de grano (RG), peso de 100 semillas (P100S) y área de capítulo (AC), en función al suelo (S), nitrógeno (N) y Biofertilizante (BIO).

Table 2. Total biomass (BT), harvest index (IC), grain yield (RG), 100 seeds weight (P100S) and chapter area (AC), as a function of soil (S), nitrogen (N) and biofertilizer (BIO).

\begin{tabular}{|c|c|c|c|c|c|}
\hline FACTOR & $\begin{array}{c}\text { BT } \\
\mathrm{g} \mathrm{m}^{-2}\end{array}$ & $\begin{array}{l}\text { IC } \\
\%\end{array}$ & $\begin{array}{c}\text { RG } \\
\mathrm{g} \mathrm{m}^{-2}\end{array}$ & $\begin{array}{c}\text { P100S } \\
\text { g }\end{array}$ & $\begin{array}{l}\mathrm{AC} \\
\mathrm{cm}^{2}\end{array}$ \\
\hline S1 & $1286 a$ & $30 a$ & $387 a$ & $7.5 \mathrm{a}$ & $327 a$ \\
\hline S2 & $1063 \mathrm{~b}$ & $26 b$ & $306 \mathrm{~b}$ & $7.0 \mathrm{a}$ & $316 b$ \\
\hline DMS & 57.8 & 1.3 & 18.3 & 0.7 & 2.0 \\
\hline No & $967 b$ & $30 a$ & $304 b$ & $6.6 \mathrm{~b}$ & $289 \mathrm{~b}$ \\
\hline N100 & $1381 \mathrm{a}$ & $26 \mathrm{~b}$ & $390 a$ & $7.9 a$ & $354 a$ \\
\hline DMS & 89.2 & 1.0 & 18.0 & 0.1 & 24.8 \\
\hline $\mathrm{BIO}$ & $1305 a$ & $27 b$ & $373 a$ & $7.8 \mathrm{a}$ & $352 a$ \\
\hline SBIO & $1044 \mathrm{~b}$ & $29 a$ & $321 \mathrm{~b}$ & $6.7 \mathrm{~b}$ & $290 \mathrm{~b}$ \\
\hline DMS & 75.9 & 0.9 & 37.6 & 0.5 & 4.8 \\
\hline $\mathrm{CV}$ & 3.7 & 1.7 & 5.02 & 3.2 & 3.5 \\
\hline
\end{tabular}

"Letras distintas en la misma columna indican diferencias significativas, según Tukey $=0.05$. Suelo 1 (S1), pH de 7.1 y CE de $0.49 \mathrm{dS} \mathrm{m}^{-1}$, suelo 2 (S2), $\mathrm{pH}$ de $7.8, \mathrm{CE}$ de $1.75 \mathrm{dS} \mathrm{m}^{-1}$. SBIO = Sin Biofertilizante, $\mathrm{BIO}=$ Con biofertilizante; NO y N100 $=0$ y $100 \mathrm{~kg}$ de $\mathrm{N} \mathrm{ha}^{-1}$.

\section{Nitrógeno}

En respuesta al N, la BT y $\mathrm{RG}$ se incrementó en un 42 y $28 \%$ respecto a la cero fertilización. Cabe señalar, que con la aplicación $\mathrm{N}$ también se logró aumentar el P100S y AC, lo que está relacionado con un mayor rendimiento de grano. En estudios realizados por Ali et al. (2012) y Nasim et al. (2012) al evaluar diferentes niveles de nitrógeno en girasol, encontraron que conforme la fertilización fue aumentando también lo hacia la BT y el RG. Lo anterior, indica que la generación de materia seca está directamente relacionada con la disponibilidad y aprovechamiento de los nutrimentos en el suelo (nitrógeno). El nitrógeno es el elemento más requerido por las plantas, ya que representa de 1 a $5 \%$ de la biomasa total y es constituyente integral de proteínas, ácidos nucleicos, clorofilas, coenzimas fitohormonas y metabolitos secundarios (Hawkesford et al., 2012).

\section{Biofertilizante}

La inoculación del biofertilizante en el cultivo de girasol generó un incremento en la BT y RG del 25 y $16 \%$ respecto al testigo sin aplicar, respectivamente, el cual está relacionado con el aumento en el AC y P100S. Dicho aumento en la BT, RG y sus componentes puede deberse a una mayor área radical e incremento en actividad de esta, como la liberación de exudados hormonales provocados por la aplicación de Azospirillum sp. y Glomus sp. (Aguilar et al., 2015; Espinosa et al., 2019; Venegas et al., 2019). Ali et al. (2012), observaron que al suministrar biofertilizante (Azotobacter sp. y Azospirillum sp.) en el cultivo de girasol se logró incrementar la biomasa total y rendimiento de grano en $10 \%$ respecto a la nula biofertilización. Esto resultados corroboran el hecho de que el uso de microorganismos (biofertilizante) estimula a la planta para promover una mayor producción en el cultivo de girasol.

\section{Interacción Suelo x Nitrógeno}

En general los dos tipos de suelo presentaron los valores de BT y RG más alta con la aplicación de $100 \mathrm{~kg} \mathrm{ha}^{-1} \mathrm{de}$ nitrógeno en comparación a $0 \mathrm{~kg}$ de ha-1 de nitrógeno, siendo este último donde se obtuvo la producción más baja (Figura 3). Al respecto Rasool et al. (2013), al evaluar diferentes niveles de nitrógeno lograron aumentar la BT y RG, tendencias

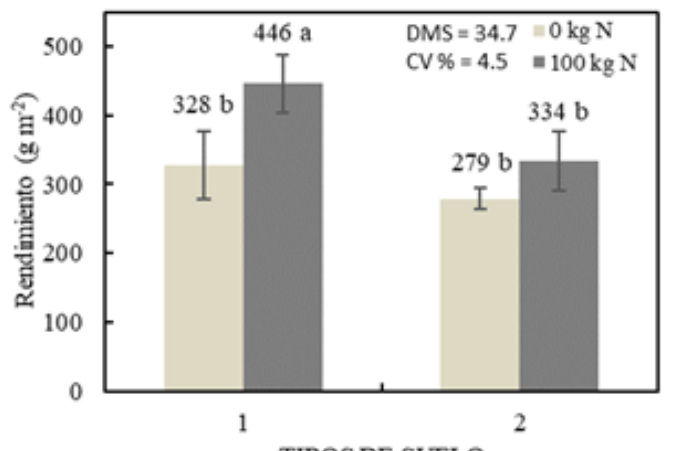

TIPOS DE SUELO

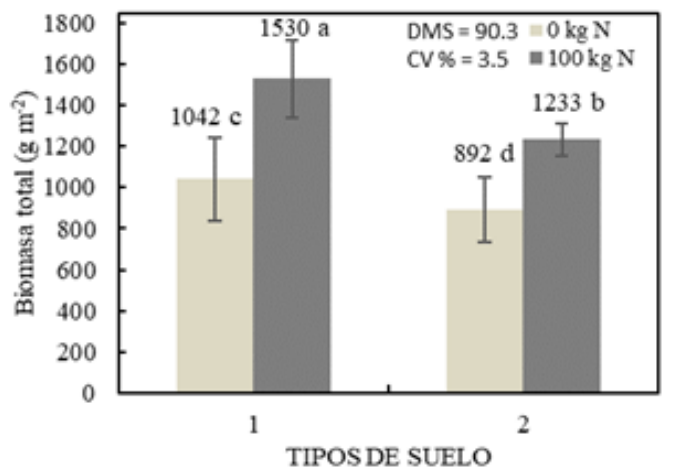

Figura 3. Biomasa total y rendimiento de grano en función del suelo y nitrógeno $(\mathrm{N})$. Letras distintas en las columnas indican diferencias significativas, según Tukey $=0.05$. Suelo $1: \mathrm{pH}$ de 7.1 y CE de $0.49 \mathrm{dS} \mathrm{m}^{-1}$, suelo $2: \mathrm{pH}$ de 7.8, CE de $1.75 \mathrm{dS} \mathrm{m}^{-1}$.

Figure 3. Total biomass and grain yield as a function of soil and nitrogen (N). Different letters on columns indicate significant differences, according to Tukey $=0.05$. Soil 1: $\mathrm{pH}$ of 7.1 and CE of $0.49 \mathrm{dS} \mathrm{m}^{-1}$, soil 2: $\mathrm{pH}$ of 7.8, CE of $1.75 \mathrm{dS} \mathrm{m}^{-1}$. 
similares al presente estudio. El suelo que presentó la mayor producción de BT y RG fue $\mathrm{S} 1 \mathrm{pH}$ de 7.1 y CE de 0.49 dS $\mathrm{m}^{-1}$ ) con la aplicación de $100 \mathrm{~kg} \mathrm{ha}^{-1}$ de nitrógeno, donde el incremento fue de 71 y $60 \%$, respecto a la nula aplicación de nitrógeno y suelo con mayor salinidad como el de $\mathrm{S} 2 \mathrm{pH}$ de 7.8 y $\mathrm{CE}$ de $1.75 \mathrm{dS} \mathrm{m}^{-1}$ ), el cual registró la menor BT y RG. Lo anterior, se debió a que los niveles altos de salinidad disminuyen los parámetros de crecimiento y producción agrícola, debido a la disminución de la actividad fotosintética, modificaciones morfológicas, estructurales y metabólicas en las plantas (Conus et al., 2009; Dos-Santos et al., 2017).

\section{Interacción Suelo x Biofertilizante}

En la interacción S x BIO (Figura 4), se puede observar que con el uso del biofertilizante se logró aumentar la producción de biomasa total y rendimiento de grano, siendo más evidente en S1, en donde el incremento fue del 25 (BT) y $20(R G) \%$ respecto a S2. El uso de biofertilizante posiblemente estimuló la producción de sustancias promotoras del crecimiento (hormonas y enzimas), lo cual generó un incremento en la biomasa de la planta de girasol como lo indica Ahmed y El-Araby (2012). Cabe señalar, que S1 presenta un suelo con pH neutro ( $\mathrm{pH}$ de 7.1) y CE de $0.49 \mathrm{dS} \mathrm{m}^{-1}$, condiciones posiblemente óptimas para el desarrollo de la planta, ya que el aumento en la salinidad produce una reducción en
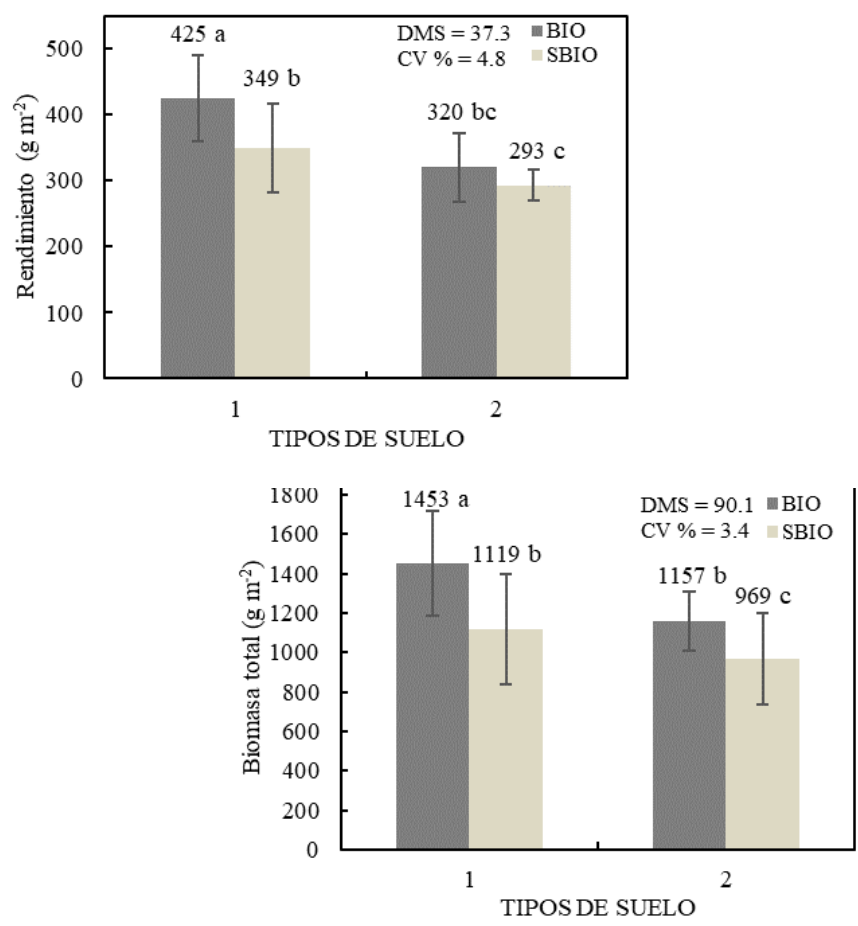

Figura 4. Biomasa total y rendimiento de grano en función del suelo y biofertilizante (BIO) (Con y Sin Biofertilizante). Letras distintas en las columnas indican diferencias significativas, según Tukey $=0.05$. Suelo $1: \mathrm{pH}$ de $7.1 \mathrm{y}$ CE de $0.49 \mathrm{dS} \mathrm{m}^{-1}$, suelo 2: $\mathrm{pH}$ de $7.8, \mathrm{CE}$ de $1.75 \mathrm{dS} \mathrm{m}^{-1}$.

Figure 4. Total biomass and grain yield as a function of soil and biofertilizer (BIO) (With and without Biofertilizer). Different letters on columns indicate significant differences, according to Tukey $=0.05$. Soil 1: $\mathrm{pH}$ of 7.1 and CE of $0.49 \mathrm{dS} \mathrm{m}^{-1}$, soil 2: $\mathrm{pH}$ of $7.8, \mathrm{CE}$ of $1.75 \mathrm{dS} \mathrm{m}^{-1}$. la BT y RG, como se pudo observar al comparar S1 contra S2. Por lo que, la reducción del crecimiento de las plantas está relacionada con el exceso de acumulación de ciertos iones en los tejidos vegetales, causado por una posible toxicidad iónica (salinidad) e inestabilidad nutricional en el suelo (DosSantos et al., 2017).

\section{Interacción Nitrógeno $\mathrm{x}$ biofertilizante}

Para la combinación de N x BIO (Figura 5), se observó que al aplicar de $100 \mathrm{~kg} \mathrm{ha}^{-1}$ de nitrógeno la producción de biomasa total y rendimiento de grano se incrementó en un 57 y $28 \%$ respecto a la nula fertilización, al incorporar el biofertilizante en los tratamientos con nitrógeno, se logró que la biomasa total y rendimiento de grano aumentara en un 21 y $26 \%$ más, respecto a la fertilización de $100 \mathrm{~kg} \mathrm{ha}^{-1}$ de nitrógeno, valores semejantes registraron Jalilian et al. (2012), donde indican que con la aplicación del biofertilizante se promueve el crecimiento de la planta de girasol, lo que genera una mayor biomasa total y rendimiento de grano. Por su parte Madhurendra et al. (2009), mencionan que con la aplicación de $100 \mathrm{~kg}$ de $\mathrm{N}$ ha ${ }^{1}+$ Azospirillum y Azotobacter se eleva el rendimiento de grano en comparación a solo la fertilización nitrogenada.

\section{Interacción Suelo x Nitrógeno x Biofertilizante}

En la interacción $\mathrm{S} \times \mathrm{N} \times \mathrm{BIO}$ (Tabla 3), se percibió que la mayor BT y RG se registró con la aplicación de $\mathrm{N}(100 \mathrm{Kg}$ $\mathrm{ha}^{-1}$ ) y biofertilizante, siendo más evidente en el Suelo 1, ya
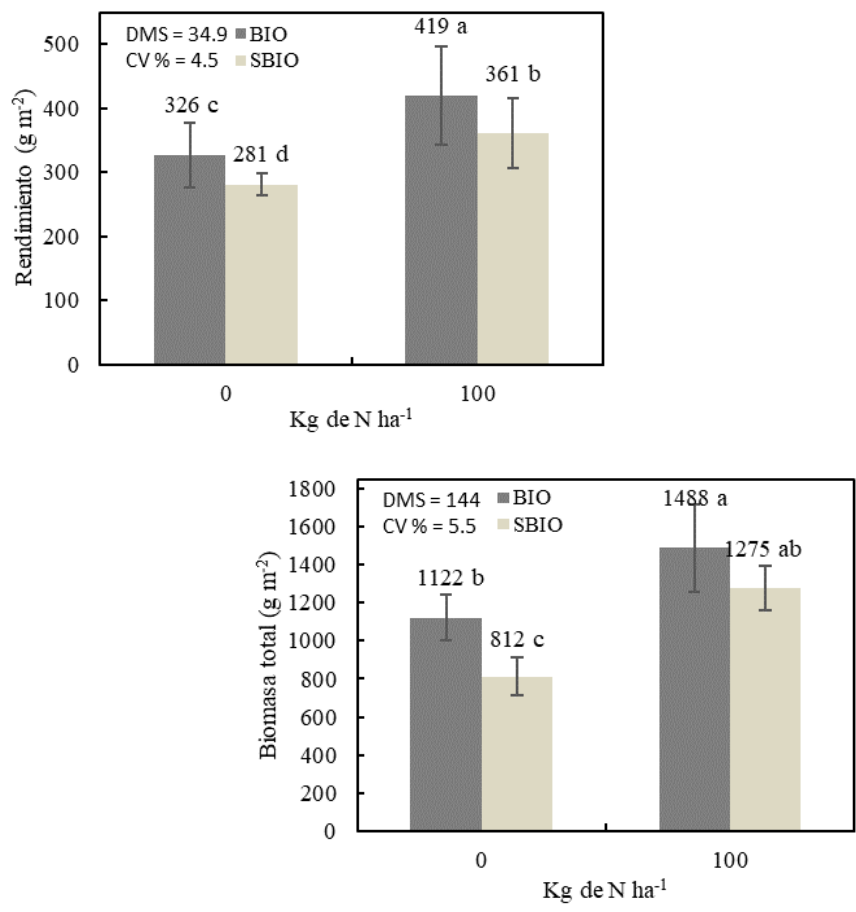

Figura 5. Biomasa total y rendimiento de grano en función del nitrógeno (N) y Biofertilizante (BIO) (Con y Sin Biofertilizante). Letras distintas en las columnas indican diferencias significativas, según Tukey $=0.05$.

Figure 5. Total biomass and grain yield as a function of nitrogen $(\mathrm{N})$ and Biofertilizer (BIO) (With and without Biofertilizer). Different letters on columns indicate significant differences, according to Tukey $=0.05$. 
Tabla 3. Biomasa total (BT), índice de cosecha (IC), rendimiento de grano (RG), peso de 100 semillas (P100S) y área de capítulo (AC), en función al suelo, nitrógeno (N) y Biofertilizante (BIO).

Table 3. Total biomass (BT), harvest index (IC), grain yield (RG), 100 seeds weight (P100S) and chapter area (AC), as a function of soil, nitrogen (N) and biofertilizer (BIO).

\begin{tabular}{|c|c|c|c|c|c|c|c|}
\hline SUELO & $\begin{array}{c}\mathbf{N} \\
\mathrm{Kg} \mathrm{ha}^{-1} \\
\end{array}$ & $\begin{array}{c}\text { Bio } \\
\text { fertilizante } \\
\end{array}$ & $\begin{array}{c}\text { BT } \\
\mathrm{g} \mathrm{m}^{-2} \\
\end{array}$ & $\begin{array}{l}\text { IC } \\
\% \\
\end{array}$ & $\begin{array}{c}\text { RG } \\
\mathrm{g} \mathrm{m}^{-2} \\
\end{array}$ & $\begin{array}{c}\text { P100S } \\
\text { G }\end{array}$ & $\begin{array}{l}\text { AC } \\
\mathrm{cm}^{2}\end{array}$ \\
\hline \multirow{4}{*}{1} & \multirow{2}{*}{0} & $\mathrm{BIO}$ & $1215 b$ & $28 \mathrm{~b}$ & $339 a$ & $7 a$ & $310 c$ \\
\hline & & SBIO & 869 bc & $29 a b$ & $287 a$ & $6 a$ & $271 \mathrm{e}$ \\
\hline & \multirow{2}{*}{100} & $\mathrm{BIO}$ & $1611 \mathrm{a}$ & $23 c$ & $482 \mathrm{a}$ & $9 a$ & $398 a$ \\
\hline & & SBIO & $1368 \mathrm{ab}$ & $25 c$ & $411 \mathrm{a}$ & $8 a$ & $318 c$ \\
\hline \multirow{4}{*}{2} & \multirow{2}{*}{0} & $\mathrm{BIO}$ & $1029 b c$ & $30 a$ & $284 a$ & $6 a$ & $308 d$ \\
\hline & & SBIO & $756 c$ & $32 \mathrm{a}$ & $255 a$ & $6 a$ & $257 f$ \\
\hline & \multirow{2}{*}{100} & $\mathrm{BIO}$ & $1285 \mathrm{ab}$ & $28 \mathrm{~b}$ & $357 a$ & $8 a$ & $385 b$ \\
\hline & & SBIO & $1181 \mathrm{abc}$ & $30 a$ & $311 \mathrm{a}$ & $7 a$ & $315 \mathrm{~cd}$ \\
\hline
\end{tabular}

"Letras distintas en la misma columna indican diferencias significativas, según Tukey = 0.05. Suelo 1: $\mathrm{pH}$ de 7.1 y CE de $0.49 \mathrm{dS} \mathrm{m}^{-1}$, suelo 2: $\mathrm{pH}$ de 7.8, $\mathrm{CE}$ de $1.75 \mathrm{dS} \mathrm{m}^{-1}$. SBIO = Sin Biofertilizante, $\mathrm{BIO}=$ Con biofertilizante.

que la producción fue 20 y $26 \%$ mayor a la del Suelo 2 . El RG más alto está relacionado con el P100S y AC, componentes que mostraron un efecto positivo con el uso del biofertilizante en condiciones menos salinas (Prabhaharan et al., 2019). Cabe indicar, que el biofertilizante se vio favorecido por las condiciones del suelo en las que se desarrolló el cultivo. Esto señala, el hecho de que suelos con una mayor salinidad limitan el crecimiento de las plantas, incluso podría causar la muerte de está. Los efectos estimulantes de los microorganismos están relacionados con la producción de fitohormonas, que pudieran estimular la absorción de nutrientes y agua, lo que conduce a un mayor crecimiento y rendimiento de las plantas (Madhurendra et al., 2009; Jalilian et al., 2012). En lo que respecta al IC, tanto el Suelo 1 como en el Suelo 2, la aplicación de solo el nitrógeno $\left(100 \mathrm{~kg} \mathrm{ha}^{-1}\right)$ generó una mayor eficiencia en la acumulación de materia seca hacia el grano, ya que presentó los mayores porcentajes. Tendencias similares obtuvieron Aguilar et al. (2017) en el cultivo de maíz y encontraron que donde se aplicó nitrógeno el IC era más alto en comparación a las asociaciones entre nitrógeno y biofertilizantes.

\section{Rentabilidad económica}

En el Tabla 4, se presenta el análisis económico sobre la comercialización para el rendimiento de grano en el cultivo de girasol. Tanto en el suelo 1, como en el suelo 2 se observó que con la aplicación de $100 \mathrm{~kg} \mathrm{ha}^{-1}$ de nitrógeno en mezcla con el biofertilizante se obtuvo el costo total más alto, mismo que se amortizó con el ingreso total; de tal manera que generó el mayor ingreso neto y GPI, ya que por cada peso invertido en la producción de girasol se recuperó $\$ 5.80$ y $\$$ 4.00 , en el suelo 1 y 2 , respectivamente. La ganancia por peso invertido más baja se obtuvo en el suelo 2 con la aplicación de $100 \mathrm{~kg}$ de $\mathrm{N} \mathrm{ha}{ }^{-1}$ y sin biofertilizante que fue de $\$ 3.50$, sin embargo, al realizar la aplicación del biofertilizante, se logra
Tabla 4. Rendimiento de grano (RG), ingresos totales (IT), costos fijos (CF), costos variables (CV), costos totales (CT), ingresos netos (IN) y ganancia por peso invertido (GPI) en girasol en función al suelo, biofertilizante (BIO) y nitrógeno $(\mathrm{N})$.

Table 4. Grain yield (RG), total income (IT), fixed costs (CF), variable costs $(\mathrm{CV})$, total costs (CT), net income (IN) and invested peso gain (GPI) as a function of soil, biofertilizer (BIO) and nitrogen (N), in the sunflower crop.

\begin{tabular}{cccccccccc}
\hline SUELO & $\begin{array}{c}\mathbf{N} \\
\mathbf{k g} \\
\mathbf{h a}^{-1}\end{array}$ & BIO & $\begin{array}{c}\text { RG } \\
\mathbf{K g} \\
\mathbf{h a}^{-1}\end{array}$ & $\begin{array}{c}\text { IT } \\
\mathbf{\$}\end{array}$ & $\begin{array}{c}\text { CF } \\
\mathbf{\$}\end{array}$ & $\begin{array}{c}\mathbf{C V} \\
\mathbf{\$}\end{array}$ & $\begin{array}{c}\mathbf{C T} \\
\mathbf{\$}\end{array}$ & $\begin{array}{c}\text { IN } \\
\mathbf{\$}\end{array}$ & $\begin{array}{c}\text { GPI } \\
\mathbf{\$}\end{array}$ \\
\hline 1 & 0 & $\mathrm{BIO}$ & 3690 & 73800 & 9600 & 2000 & 11600 & 62200 & 5.30 \\
1 & 0 & SBIO & 2870 & 57400 & 9600 & 1600 & 11200 & 46200 & 4.10 \\
1 & 100 & BIO & 4820 & 96400 & 9600 & 4560 & 14160 & 82240 & 5.80 \\
1 & 100 & SBIO & 4110 & 82200 & 9600 & 4160 & 13760 & 68440 & 4.90 \\
\hline 2 & 0 & BIO & 2840 & 56800 & 9600 & 2000 & 11600 & 45200 & 3.80 \\
\hline 2 & 0 & SBIO & 2750 & 55000 & 9600 & 1600 & 11200 & 43800 & 3.90 \\
\hline 2 & 100 & BIO & 3570 & 71400 & 9600 & 4560 & 14160 & 57240 & 4.00 \\
\hline 2 & 100 & SBIO & 3110 & 62200 & 9600 & 4160 & 13760 & 48440 & 3.50 \\
\hline
\end{tabular}

$\mathrm{IT}=\mathrm{RG} \times$ precio por $\mathrm{kg}$ de girasol $(\$ 20.00)$. CF $=$ incluye costo de preparación del terreno, siembra, riego, manejo de maleza y plagas. CV = incluye el costo del biofertilizante y fertilizante inorgánico.

incrementar la GPI en el suelo 2 donde presenta una mayor salinidad en comparación al suelo 1. Al respecto, Escalante et al. (2007) al evaluar diferentes niveles de nitrógeno en girasol observaron una tendencia a incrementar la GPI, a medida que se aumentaba la fertilización. Por lo que, se recomienda la aplicación de nitrógeno en asociación con biofertilizante, alternativa que genera un mayor ingreso neto en el cultivo de girasol.

\section{CONCLUSIONES}

Para los suelos en estudio se encontró que la fertilización nitrogenada y el biofertilizante no modificaron el tiempo a ocurrencia de las etapas fenológicas, pero sí se observó un cambio en la biomasa total y rendimiento de grano, donde la mayor producción se registró con la aplicación de nitrógeno a $100 \mathrm{~kg} \mathrm{ha}^{-1}$ y la inoculación del biofertilizante, principalmente en las condiciones con menor salinidad. El mayor ingreso neto se logró con la aplicación de $100 \mathrm{~kg} \mathrm{ha}^{-1}$ de nitrógeno y biofertilizante, en el suelo con la salinidad más baja.

\section{REFERENCIAS}

Aguilar, C.C., Escalante, E.J.A.S., Aguilar, M.I., Mejía, C.J.A., Conde, M.V.F. y Trinidad, S.A. 2015. Rendimiento y rentabilidad de maíz en función del genotipo, biofertilizante y nitrógeno, en clima cálido. Tropical and subtropical Agroecosystems, 18: 151-163.

Aguilar, C.C., Escalante, E.J.A.S., Aguilar, M.I. y Pérez, R.A. 2017. Crecimiento, rendimiento y rentabilidad del maíz VS-535 en función del biofertilizante y nitrógeno. Ecosistemas y Recursos Agropecuarios. 4: 475-483.

Ahmed, H.F.S. y El-Araby, M.M.I. 2012. Evaluation of the influence of nitrogen fixing, phosphate solubilizing and potash mobilizing biofertilizers on growth, yield, and fatty acid constituents of oil in peanut and sunflower. African Journal of Biotechnology. 11: 10079-10088. 
Ali, N., Teymur, K. y Mona, S. 2012. Effects of bio and chemical nitrogen fertilizer on grain and oil yield of sunflower (Helianthus annuus L.) under different rates of plant density. Annals of Biological Research. 3: 1125-1131.

Bueno, J.J.E., Alonso, L.A., Volke, H.V., Gallardo, L.F., Ojeda, R.M.M. y Mosqueda, V.R. 2005. Respuesta del papayo a la fertilización con nitrógeno, fosforo y potasio en un luvisol. Terra Latinoamericana. 23:409-415.

Conus, L.A., Cardoso, P.C., Venturoso, L.R. y Scalon, S.P.Q. 2009. Germinação de sementes e vigor de plântulas de milho submetidas ao estresse salino induzido por diferentes sais. Rev. Bras. Sementes. 31: 67-74.

Doorenbos J. y. Pruitt, W.O. 1986. Las necesidades del agua por los cultivos. 1er ed. Estudio FAO. Riego y Drenaje. Manual 24.

Dos-Santos, J.B., Marenco-Centeno, C.R., Vieira de-Azevedo, C.A., Raj-Gheyi, H., De-Lima, G.S. y De-Lira, V.M. 2017. Crecimiento del girasol (Helianthus annuus L.) en función de la salinidad del agua de riego con fertilización nitrogenada. Agrociencia. 51:649-660.

Escalante, E.L.E., Escalante, E.Y.I y Lizaga, E.C. 2007. La fertilización nitrogenada en el rendimiento del girasol en México. Agronomía Costarricense. 31: 95-100.

Escalante, E.J.A. y Rodríguez, G.M.T. 2010. Sunflower biomass distribution and seed yield in saline soil of Mexico highlands. HELIA. 33: 127-134.

Escalante-Estrada, J.A.S., Rodríguez-González, M.T. y EscalanteEstrada, Y.I. 2015. Fenología, biomasa y rendimiento de cultivares de girasol en Valles Altos. Revista Mexicana de Ciencias Agrícolas. 2: 307-311.

Espinosa, P.B, Cano, R.P., Salas, P.L., García, H.J.L., Preciado, R.P., Sáenz, M.J. y Reyes, C.J.L. 2019. Bioinoculantes y concentración de la solución nutritiva sobre la producción y calidad de tomate. Biotecnia. 21: 100-107.

Flores, J.M., Chan J.L. y Bravo, L. 1985. Fenología del maíz y fríjol en el altiplano de Zacatecas. II. Unidades calor (UC) y desarrollo fenológico. Fitotecnia. 7: 66-81.

García, E. 2004. Modificación al sistema de clasificación climática de Köppen. 4a . Edición. Instituto de Geografía. Universidad Autónoma de México.

Hawkesford, M., Horst, W., Kichey, T., Lambers, H., Schjoerring, J., Moller, I. S. y White, P. 2012. Functions of macronutrients. In: Marschner, P. (Ed.), Marschners's Mineral Nutrition of higher plants, pp 135-189. USA. Academic Press.

Ishfaq, M., Ali, A., Khaliq, A. y Yaseen, M. 2009. Allometry, agronomic traits and yield of autumn planted sunflower hybrids under varying row spacing. Pak. Journal Agriculture Science. 46: 248-257.

Jalilian, J., Modarres, S.S.A.M., Farhad, S.S. y Sadat A.K. 2012. Effects of the combination of beneficial microbes and nitrogen on sunflower seed yields and seed quality traits under different irrigation regimes. Field Crops Research. 127: 26-34.

Martínez, V.N., López, A.C.V., Basurto, S.M. y Pérez, L.R. 2011. Efectos por salinidad en el desarrollo vegetativo. Medio Ambiente y Desarrollo Sustentable. 5: 156-161.

Madhurendra, Prasad, N. y Akhuri, R.K. 2009. Effect of biofertilizer on sunflower, Helianthus annuus L. Indian Journal of Oilseeds Research. 26: 167-177.
Mena, L.J.L., Díaz, P.A.L. y Valdés, H.A. 2018. Efectos de dos biofertilizantes en el desarrollo del girasol. Revista de Ciencias Agrarias. 41: 933-944.

Mishra, B.K. y Dadhich, S.K. 2010. Methodology of nitrogen biofertilizer production. Journal Adv. Dev. Research. 1: 3-6.

Morales, R.E.J., Escalante, E.J.A., Tijerina, C.L., Volke, H.V. y Sosa, M.E. 2006. Biomasa, rendimiento, eficiencia en el uso del agua y de la radiación solar del agrosistema girasol-frijol. Terra Latinoamericana. 24: 55-64.

Nasim, W., Ahmad, A., Bano, A., Olatinwo, R., Usman, M., Khaliq, T., Wajid, A., Hammad, H. M., Mubeen, M. y Hussain, M. 2012. Effect of Nitrogen on Yield and Oil Quality of Sunflower (Helianthus annuus L.) Hybrids under Sub Humid Conditions of Pakistan. American Journal of Plant Sciences. 3: 243-251.

Prabhaharan, R., Borboa, F.J., Rosas, B.E.C., Cárdenas, L.J.L., Ortega, G.J. y Rueda, P.E.O. 2019. Phaseolus acutifolius associated with Bacillus amyloliquefaciens and Azospirillum halopraeferens under salinity conditions. Biotecnia. 21: 127132.

Rasool, F.U., Hassan, B. y Jahangir, I.A. 2013. Growth and yield of sunflower (Helianthus annus L.) as influenced by nitrogen, sulphur and farmyard manure under temperate conditions. SAARC Journal of Agriculture. 11: 81-89.

Sadras, V.O. y Hali, A.J. 1988. Quantification of temperature, photoperiod and population effect on plant leaf area in sunflower crop. Field Crop Research. 18: 185-196.

SAS Institute. 2004. SAS User's Guide. Statistics. SAS Institute. Cary, NC, USA.

Schneiter, A. y Miller, J.F. 1981. Description of sunflower growth stages. Crop Science. 21: 901-903.

SIAP (Servicio de Información Agropecuaria y Pesquera) [Consultado 10 diciembre 2018] 2018. Producción agrícola de cultivos cíclicos y perennes. Disponible en: www.siap. gob.mx

Sotelo, C.E., Zalocar, Y., Cándida, I.M., Collavino, M., Grasso, D., Cassan, F. 2016. Biofertilización con cianobacterias (Nostoc sp.). Efecto sobre el rendimiento del girasol (Helianthus annuus L.), en suelos del Chaco. Agrotecnia. 23: 21.

Tapia, G.J.J., Ferrera, C.R., Varela, F.L., Rodríguez, O.J.C., Soria, C.J.C., Tiscareño, I.M.A., Loredo, O.C., Alcalá, J.J. y Villar, M.C. 2010. Infectividad y efectividad de hongos micorrízicos arbusculares nativos de suelos salinos en el cultivo de lechuga (Lactuca sativa). Revista Mexicana de Micología. 31: 69-74.

Venegas, G.J., Méndez, I.C., Martínez, M.E.K., Ceja, T.L.F. y Rodríguez, T.M.D. 2019. Producción orgánica de Beta vulgaris subespecie cicla con inoculantes microbianos. 21: 121-126.

Werteker, M., Lorenz, A., Johannes, H., Berghofer, E. y Findlay, C.S. 2010. Environmental and Varietal Influences on the Fatty Acid Composition of Rapeseed, Soybeans and Sunflowers. J. Agronomy \& Crop Science. Austrian Agency for Health and Food Safety. 196: 20-27.

Zarabi, M., Alahdadi, I., Akbari, G.A. y Akbari, G.A. 2011. A study on the effects of different biofertilizer combinations on yield, its components and growth indices of corn (Zea mays L.) under drought stress conditions. African Journal Agriculture Research. 6: 681-685. 